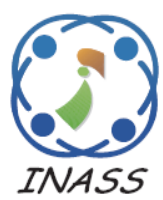

\title{
Automated False Positive Reduction and Feature Extraction of Kidney Stone Object in 3D CT Images
}

\author{
Nilar Thein ${ }^{1 *}$ \\ Teguh Bharata Adji ${ }^{1}$ \\ Kazuhiko Hamamoto ${ }^{2}$ \\ Hanung Adi Nugroho ${ }^{1}$ \\ ${ }^{I}$ Department of Electrical Engineering and Information Technology, Universitas Gadjah Mada, Indonesia \\ ${ }^{2}$ Department of Information Media Technology, Tokai University, Tokyo, Japan \\ * Corresponding author's Email: nilarthein.mti13@mail.ugm.ac.id
}

\begin{abstract}
CT imaging is widely used for a variety of diagnostic and therapeutic purposes. It is a best effective and efficient tool in kidney stones diagnostic and treatment strategies whenever contactless measurements of quality are necessary to examine different types of stones. Accurate segmentation is a key process in CT diagnostic imaging. But, there are some challenges to distinguish among stones, stents or nephrostomy tubes, calcification and bone fragments in stone diagnostic CT imaging. This study proposed and experimentally investigated a scheme to reduce false positive for stone detection and to extract meaningful structural information for diagnosis of kidney stones. Firstly, the interested object (stone) is segmented by Otsu's thresholding method and morphological operation. Secondly, the parameters based on 3D morphological features are measured for stone detection. In false positive reduction, the unwanted disturbances (bone fragments, image noise, calcification and stents) are eliminated using novel volume-ratio based thresholding and SAV ratio based thresholding. Finally, it provided an output that identifies meaningful structural information (Surface-area-to-volume ratio, volume, location and density of kidney stone) for kidney stone diagnosis. Digitized transverse abdomen CT images from 35 patients with kidney stone cases were statistically analyzed and validated. The estimation of coordinate points in the stone region was measured independently by the expert radiologists to get the validated data for the analysis. The proposed algorithm was significantly reduced the false positives with $70 \%$ of overall accuracy. Moreover, it can present the meaningful information of the kidney stone. This may lead to more accurate stone management.
\end{abstract}

Keywords: Feature extraction, SAV ratio based thresholding, Volume-ratio based thresholding.

\section{Introduction}

The prevalence of kidney stone disease is a common and increasing problem across the world. Kidney stone disease can seriously affect health and quality of life and can result in a kidney dead or kidney function failure. According to the survey in 2009 , there were approximately 1.3 million people of cases of kidney stone disease in the United States. Fortunately, most of kidney stone disease will recover by providing right treatment decision and by preventing stone recurrence.

Kidney stone can be detected by using both traditional diagnostic tests (urine testing, blood testing and biopsy testing) and imaged-based test (ultrasound testing, CT testing and MRI testing).
According to the consideration based on time taken, dependency on human experts, cost and sensitivity in diagnostic testing, CT and ultrasound imaging are very essential and helpful modality in kidney stone diagnosing. But, in ultrasound imaging, there is no ability to view the interior of bony structures because ultrasound images have been produced using the intensity of the reflected high-frequency sound wave. Moreover, ultrasound imaging for obese patients may experience a less effective because deeper and deeper it passes through fat and tissue, weaker and weaker its clarity be. The accuracy of ultrasound imaging relies on a large degree on the technician skill. But, CT result offers a higher contrast image which can identify internal structure, size, density and texture and can see their shape at different angles and planes. 


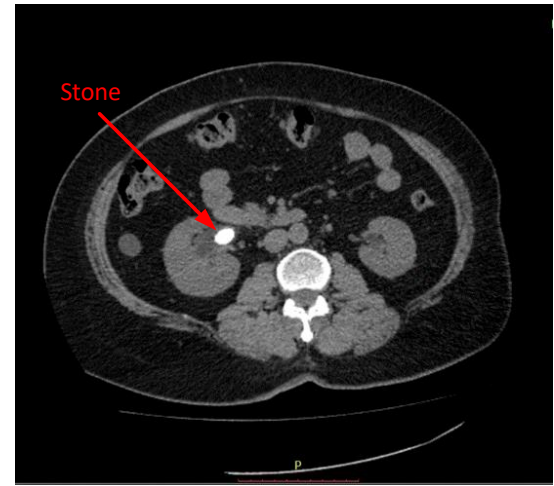

(a)

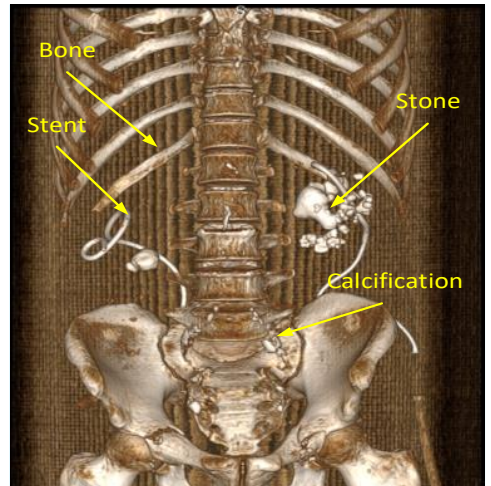

(b)

Figure. 1 Kidney stone in: (a) CT slice and (b) Stone, stent, Calcification and bones on CT in bone window

CT image can show internal body structures without overlapping structures [1]. Each 2D CT scan slice shows the shape of the stone as shown in Fig. 1 (a).

Computer aided detection on CT imaging can handle large number of cases with the same accuracy. Information gained from CT imaging is the first diagnose step in kidney stone disease management [2]. Because diagnosing on 2D slices by paging up and down is time-consuming, then in these days of radiology, 3D diagnostic imaging technology on CT data is developing to show clients' actual anatomy more clearly and accurately.

The most widespread algorithm for 3D visualization of CT data has been used for manualsegmentation and automated-segmentation techniques in pre-processing. Both these segmentation techniques have advantage and disadvantage. On the other hand, manual and semiautomatic methods are not sensitive to noise but they are tedious, require some training, poor reproducibility and need much attention to detail. On the other hand, fully automatic methods perform without training and with high-reproducibility but they are sensitive to noise and unexpected situations leading to errors [3]. Although a fair amount of research has been performing in segmentation of abdominal organs, such as the liver, kidneys, and spleen, from CT scan image, different organs and tissues have very similar gray levels, which consigns thresholding to limited utility [4]. Kidney stone segmentation on CT imaging has also difficult due to the complexity in differentiating the stone, the stent, calcification and bone which have similar gray levels [5] as shown in Fig. 1 (b). Therefore, the feature parameter without gray level values is vital for feature extraction of automate kidney stone detection.

\section{Related research}

Recently, several research works were reported to identify the kidney stone from the abdominal CT scan. Two common imaging tests to check for kidney stone are ultrasound imaging and CT scan imaging. Some of the previous studies proposed for kidney stone identification have been developed using ultrasound imaging with segmentation, feature extraction and classification methods [6 - 11]. Although these ultrasound imaging researches have been presented with good results, the algorithms applied were complex structured algorithms consisting of complex computations. Moreover, there was also some failure in certain cases. Therefore, there was a need to involve the research study using a simple and more effective novel algorithm. Hence, researches of stone detection on CT imaging were proposed [12 - 16] in Table 1.

Previous stone detection on CT imaging was conducted in preprocessing, segmentation by selecting the optimum slice, feature extraction by using 2D matrix. Therefore, accuracy of the system was relatively low and there was some failure in some cases. Moreover, there is a limitation for the stone developed in the ureter and bladder because the systems reported kidney stone extraction from the selected kidney region. To overcome this limitation, the present study is aim to develop the kidney stone detection in the urinary tract (kidneys, ureters, and bladder) without limitation the stone location.

\section{Methods and materials}

\subsection{Mathematical theory}

The proposed algorithm was designed on the application of image processing techniques and 
Table 1. Literature review for kidney stone detection on CT imaging

\begin{tabular}{|c|c|c|}
\hline & Proposed Methodology & Result \\
\hline \multirow{2}{*}{$\begin{array}{l}{[12]} \\
100 \\
\text { patient } \\
\text { dataset }\end{array}$} & \multirow{2}{*}{ 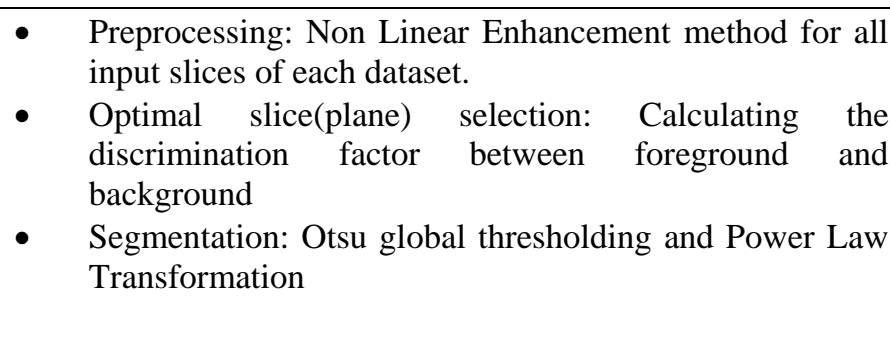 } & $\begin{array}{l}\text { - Advantage: } \\
\text { identification (present stone or } \\
\text { absent stone) and stone } \\
\text { location with } 87 \% \\
\text { identification accuracy }\end{array}$ \\
\hline & & $\begin{array}{l}\text { - Limitation: The clarity of } \\
\text { segmented output is fail in } \\
\text { some case. }\end{array}$ \\
\hline \multirow{2}{*}{$\begin{array}{l}{[13]} \\
50 \text { patient } \\
\text { dataset }\end{array}$} & \multirow{2}{*}{$\begin{array}{l}\text { - } \quad \text { Preprocessing: Crop the kidney regions in CT image by } \\
\text { regarding X and Y direction of the matrix, manually. } \\
\text { - } \text { Optimal slice(plane) selection: Fuzzy c-means clustering } \\
\text { - } \quad \text { Segmentation: Level set segmentation } \\
\text { - } \quad \text { Identification: By considering region parameter } \\
\text { extraction (centroid and area) }\end{array}$} & $\begin{array}{l}\text { - Advantage: To detect the size } \\
\text { and location of the stone with } \\
\text { low execution time }\end{array}$ \\
\hline & & $\begin{array}{l}\text { - Limitation: Segmentation } \\
\text { based on the selected slice, } \\
\text { Remaining false positive in } \\
\text { the segmented output. }\end{array}$ \\
\hline \multirow[t]{2}{*}{$\begin{array}{l}{[14]} \\
39 \text { patient } \\
\text { dataset }\end{array}$} & \multirow{2}{*}{$\begin{array}{l}\text { Preprocessing: Localization } \\
\text { Morphological operation), Contrast } \begin{array}{c}\text { (Binarization, } \\
\text { Adjustment, }\end{array} \\
\text { Intensity based thresholding, Combining the slices using } \\
\text { Logical OR operation, Connected component labeling, } \\
\text { Elimination the Spine and Ribs (by regarding a } \\
\text { coordinate point and size ratio, manually). } \\
\text { Segmentation: } \\
\text { Kidney Area segmentation: \{Restriction and Object } \\
\text { Detection (Arbitrary points and distance formula) }\} \\
\text { Kidney stone segmentation. \{Virtual Region } \\
\text { Segmentation (location of two arbitrary points, center of } \\
\text { mass, and its relative radius value) } \\
\text { Detection: Marking from the segmented output }\end{array}$} & $\begin{array}{l}\text { - Advantage: Result the } \\
\text { location of stone (Left or } \\
\text { Right), and stone size, with } \\
84.61 \% \text { accuracy. Give the } \\
\text { number of stone, area of } \\
\text { stone. }\end{array}$ \\
\hline & & $\begin{array}{l}\text { - Limitation: Still containing } \\
\text { unwanted regions in the } \\
\text { output result. In the case of } \\
\text { the irregular height abdomen, } \\
\text { the program impossible to } \\
\text { provide the correct result. }\end{array}$ \\
\hline \multirow[t]{2}{*}{$\begin{array}{l}{[15]} \\
192 \\
\text { patient } \\
\text { dataset }\end{array}$} & \multirow{2}{*}{ 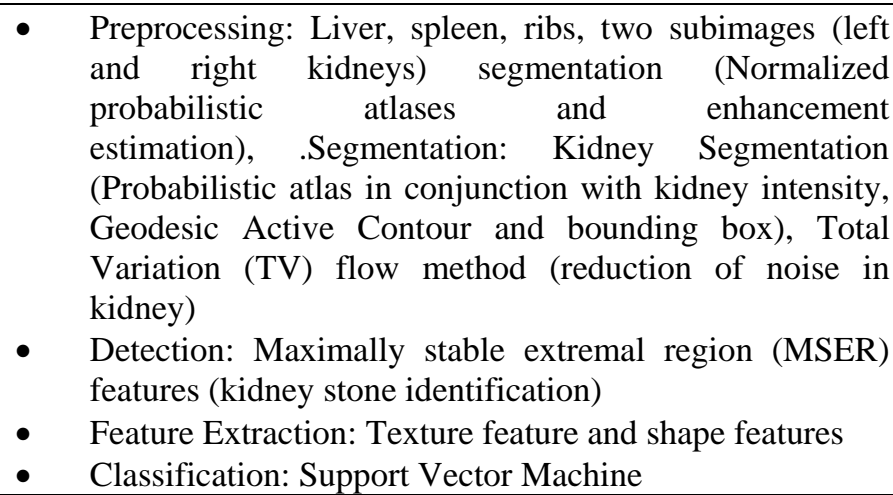 } & 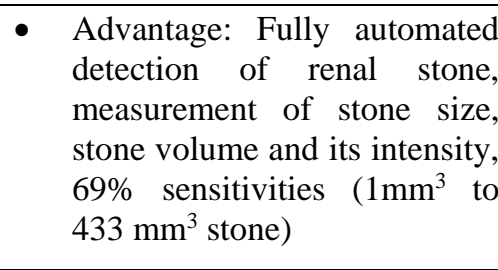 \\
\hline & & $\begin{array}{l}\text { - Limitation: Segmentation } \\
\text { accuracy was relatively low } \\
\text { because of small stone over } \\
\text { segmentation of Geodesic } \\
\text { Active Contour }\end{array}$ \\
\hline \multirow[t]{2}{*}{ [16] } & \multirow{2}{*}{$\begin{array}{l}\text { Preprocessing: Kidney region detection (using intensity } \\
\text { and location thresholding) } \\
\text { Renal mass extraction: Renal extraction from the Kidney } \\
\text { (using intensity and location thresholding) } \\
\text { False positive reduction: patch-based texture and context } \\
\text { feature } \\
\text { Renal mass segmentation: region growing, active } \\
\text { contours and outlier removal (size and shape criteria). }\end{array}$} & $\begin{array}{l}\text { - Advantage: Renal mass } \\
\text { segmentation with } 89.91 \% \\
\text { specificity, } 98.96 \% \text { specificity } \\
\text { and } 88.94 \% \text { DSC. }\end{array}$ \\
\hline & & $\begin{array}{l}\text { - Limitation: Segmentation } \\
\text { accuracy was relatively low. }\end{array}$ \\
\hline
\end{tabular}

geometry principles volume of sphere. For image pre-processing techniques, thresholding and morphological operation are applied. And, area, volume of object, surface area of object, and SAV 
ratio were measured based on the $3 \mathrm{D}$ selected kidney stone such as geometry principles. Several works on segmentation and feature extraction based on 3D morphological measurement are described in the next section.

\subsection{Image segmentation}

Image segmentation is a process of partitioning an original image into multiple regions or segments (sets of pixels), in other words, it is the classification of all pixels. In each of pixels in a region, there are some characteristics or computed properties such as color, intensity, or texture [17]. There are mainly four types of image segmentation algorithms as below:

- Edge-based segmentation,

- Region-based segmentation,

- Graph cut segmentation,

- Thresholding-based segmentation,;

Among them, thresholding-based segmentation is used in my proposed algorithm because the threshold technique is based on local pixel intensity levels. The intensity distribution between the region of foreground (stone) and background (other organs) is very distinct, a single threshold value can be applied into partition of both regions.

When the pixel of the background image differs enough with the foreground, a threshold value selecting is very easy for segmentation. It is a simplest, easiest and fastest technique among all of the existed segmentation methods. Nevertheless, it is not easy to find an appropriate threshold which can separate the image into two groups directly [18][19]. The major problem with thresholding is that- we only consider to the intensity, not the relationship between pixels. Sometimes it is occurred of losing too many regions and getting too many extraneous background pixels due to the inadequate threshold value [20].

a) Otsu's Method:

Otsu's thresholding is a global thresholding techniques using histogram of the image to search threshold value. From analyzing the distribution of gray values of an image (its histogram), two separate peaks are regarded such as one for foreground voxels and other for background voxels. Then Otsu's defines the threshold $\theta$ between these two peaks for the value minimizing the weighted sum of withinclass variances. This is equivalent to maximizing the between-class scatter. For an image taking on discrete voxel values $\mathrm{k}$ in Eq. (1), the optimal threshold is

$$
\begin{gathered}
\theta_{o t s u}=\operatorname{argmax}_{\theta}\left\{\sum_{k<\theta} p(k)\left(\mu_{0}-\mu\right)^{2}+\right. \\
\left.\sum_{k \geq \theta} p(k)\left(\mu_{1}-\mu\right)^{2}\right\}
\end{gathered}
$$

where,

$p$ is the normalized histogram

$$
\begin{aligned}
& \mu: \text { Mean }\{f(x)\} \\
& \mu_{1}: \text { Mean }\{f(x) \mid f(x) \geq \theta\} \\
& \mu_{0}: \text { Mean }\{f(x) \mid f(x)<\theta\}
\end{aligned}
$$

In this paper, we focus on kidney stone segmentation of CT by using Otsu's thresholding technique [21] and morphological operation to enhance the segmented result.

b) Morphological operation: Morphology is a broad set of shape-based image processing operations. The aim of using morphological operations is to take the imperfections away from the structure of image. Two basic operations of morphological operation are morphologic erosion and dilation: erosion typically removes small objects, and the subsequent dilation tends to restore the shape of the objects that remain. Most of operations develop on a combination of erosion and dilation process. In binary image and intensity-based image, morphological opening and morphological filling are commonly used for image segmentation [22].

Morphological opening: It is a combinational operation of erosion and dilation to give the relationship between open images morphologically. It can smooth the outline of the object, clear the narrow bridges and eliminates minor extension present in the object.

Morphological filling: Morphological filling is one of the easiest methods to fill holes in a closed boundary (ROI). In this syntax, a hole is a set of background pixels that cannot be reached by filling in the background from the edge of the image.

Matlab toolbox function "imfill" performs this computation automatically together using the optional argument "holes". Morphological filling can be a smooth of the surface of the segmented object.

\subsection{D shape feature extraction}

Feature extraction is a technique to extract the relevant information of the image to solve the computational tasks. Several features based on 3D voxel-based morphology are computed from the segmented kidney stone as described below:

a) Area 
Area of object is a basic parameter in 3D shape feature extraction. Area of a kidney stone is specified by counting the number of voxels in the specific region [23].

$$
\begin{aligned}
& \text { Area }(A)= \\
& \quad \text { Number of voxels in object }
\end{aligned}
$$

b) Volume of stone

For 3D shape of kidney stone, volume estimation is more useful and accurate than stone diameter measurement [24]. Volume of a stone is computed by multiplying the area of $3 \mathrm{D}$ stone with voxel resolution (voxel spacing).

$$
\text { Volume }(V)=\text { Area } \times \text { resolution }
$$

\section{c) Surface Area of stone}

Surface area of stone is one of the important factors to access the stone burden. Surface area of the 3D binary structure is estimated by image resolution that is a 1-by-3 row vector containing resolution in the $\mathrm{X}, \mathrm{Y}$ and $\mathrm{Z}$ direction (in that order). This surface area calculation is proved by comparing with theoretical value of surface area of sphere $\left(4 \pi r^{2}\right)$ in [25].

$$
S A=\sqrt{\left(x-r e s_{x}\right)^{2}+\left(y-r e s_{y}\right)^{2}+\left(z-\operatorname{res}_{z}\right)^{2}}
$$

Where, SA: surface area of stone

$r e s_{x}, r e s_{y}, r e s_{z}$ : resolution of voxel in the $\mathrm{X}, \mathrm{Y}$ and $\mathrm{Z}$ direction

d) Volume of sphere

Mathematically, volume of a sphere is calculated based on its radius as following equation.

$$
\text { Volume of sphere: } V=\frac{4}{3} \pi r^{3}
$$

Where, V: volume of sphere

r: radius of sphere

Because most of kidney stone have asymmetric-shape, volume estimation using surface area of stone is more reasonable than estimation using radius. The relationship between radius and surface area of sphere:

$$
\text { Surface Area }(S A)=4 \pi r^{2}
$$

Therefore, the volume of a sphere is formulated as follow:

Volume of sphere: $V=\sqrt{\frac{(S A)^{3}}{36 \pi}}$

Where, V: volume of sphere

SA: surface area of sphere

e) SAV (Surface-area-to-volume ratio)

In [26], the consequence of the isoperimetric inequality in $3 \mathrm{D}$ object is related with SAV ratio, the amount of surface area per unit volume of an object. SAV ratio enables to estimate the shape of 3D object. Larger the gap between the SAV of objects be, more different the shape of objects have. Spherical shaped object has the smallest SAV while tetrahedron, triangular shaped object, has the largest SAV. As a standard, SAV ratio of a spherical shaped object is calculated as follow.

$$
S A V=\frac{3}{r}
$$

Where r: radius of sphere

But, volume of the object, instead of radius, is also used for SAV calculation. Therefore, SAV ratio can be formulated as follow:

$$
S A V_{\text {sphere }}=\frac{4.836}{\sqrt[3]{V}}
$$

Where: SAV: the surface area of stone

$$
\mathrm{V} \text { : the volume of stone }
$$

Moreover, SAV ratio can also be estimated by using surface area and volume of the segmented stone.

$$
S A V_{\text {stone }}=\frac{\text { Surface area of stone }}{\text { Volume of stone }}
$$

f) Ratio Volume Sphere (RVS)

To predict the average kidney stone-shape, 3Dreconstructed stone volumes was compared with the volume of spheroid using diameter of stones [27]. In [28], 3D ROI manager generated ratio volume ellipsoid (RVE) for branching analysis.

RVS is the ratio of the volume of the test object to the volume of the sphere with the same surface area of the test object. As most of stone is irregular shape structure and some has branches, volume of the segmented stone is greater than volume of sphere using the same surface area. Therefore, RVS of no-branches 


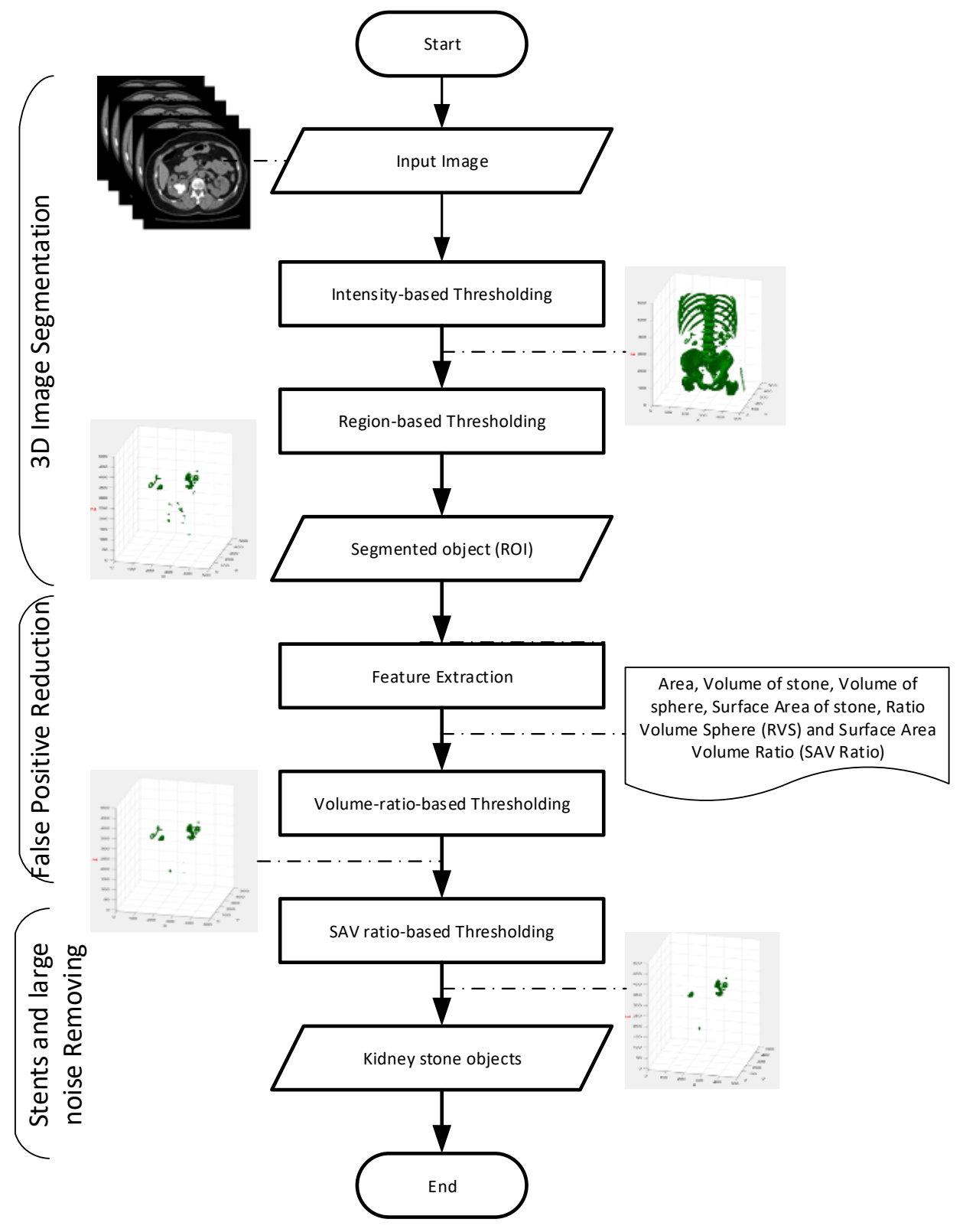

Figure. 2 The proposed 3D kidney stone segmentation and false positive reduction

stone is between 0 and 1, RVS of branches stone is greater than 1 .

$$
R V S=\frac{V_{o b j}}{V_{s p h}}
$$

Where: $V_{o b j}$ : Volume of a test object (the selected stone)

$V_{s p h}:$ Volume of a sphere with the same surface area as test object.

\subsection{Dataset Preparation}

The digitized transverse abdomen CT scans images from 35 patients with kidney stone cases were taken with Toshiba Aquilion 64-slice CT Scanner and obtained from No_2, Military Hospital, Yangon. The number of slices for each patient has between 500 slices and 600 slices with variable kidney stone conditions through their CT scan. The dataset applied in this experimental research contains various renal stone cases with calcification, stent and without limitation in stone location. The degree of accuracy and efficiency of the proposed algorithm has been proved by the estimation of coordinate points in the stone region by the expert radiologists. Moreover, the output clarity of the proposed algorithm has been compared with the result of existing research and commercial software (RadiAnt DICOM Viewer). 


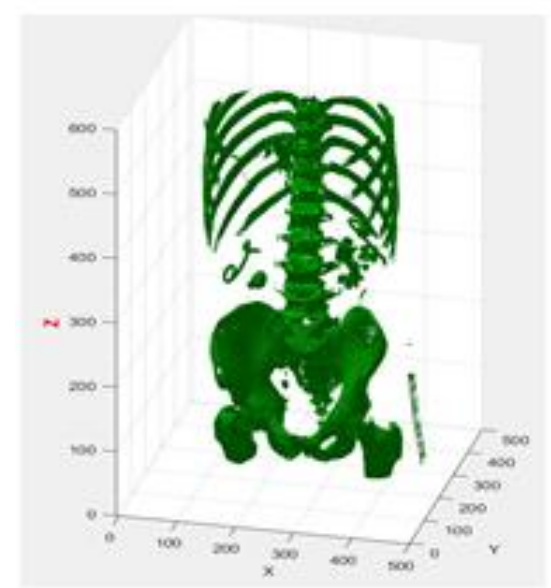

(a)

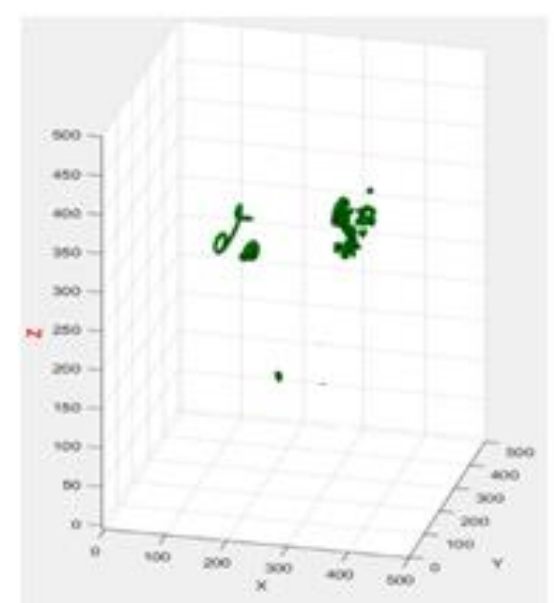

(c)

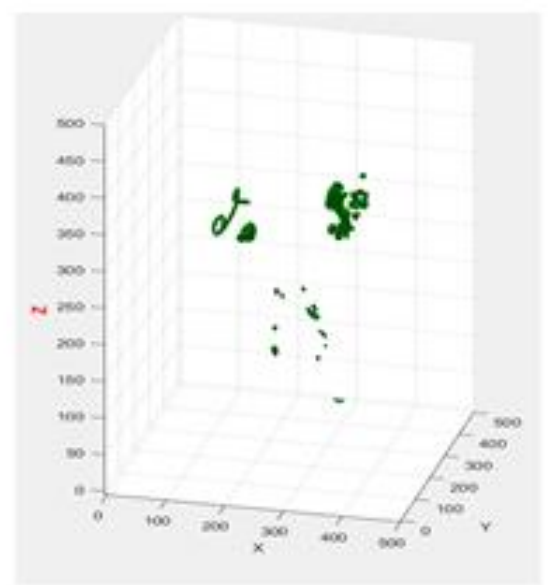

(b)

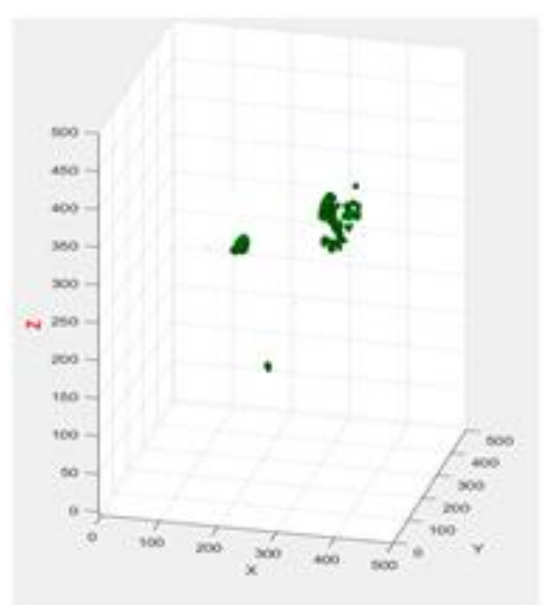

(d)

Figure. 3 The experimental result for kidney stone segmentation and false positive reduction (using the proposed algorithm): (a) segmented result after low intensity region removing, (b) segmented result after large region removing,

(c) segmented result after false positive reduction, and (d) segmented result after stent removing,

\subsection{Proposed Method}

This paper describes automate noise removal and feature extraction techniques with the help of thresholding technique and 3D morphology feature extraction from the segmented 3D object as shown in Fig. 2. These were applied interactively in the proposed scheme to develop three levels of image analyses (segmentation, false positive reducing and stent and large noise removing) to enhance the kidney stone segmentation. Firstly, all slices from each patient were inputted into the program and then the unwanted regions were taken away from CT scan image in order to segment the kidney stone without losing the required information. Next, the remained noise in the segmented output was also removed to reduce false positive. Finally, the proposed algorithm were analyzed all remained object whether is kidney stone or non-stone in large noise removing. If there is the tube or large noise, the algorithm will eliminate these objects from the image.

\section{Result and discussion}

\subsection{Implementation detail}

In this paper, a software implementation for image segmentation and false positive reduction based on Otsu's thresholding, morphological operation and 3D morphological feature extraction is proposed as follows. The required threshold values for each process are obtained from prior knowledge of CT scan image and feature extraction of 3D segmented object in Table 2.

\subsection{Kidney stone segmentation (pre-processing)}

Pre-processing stage consists of noise removal scheme using Otsu's thresholding and morphological operation. Loading the input DICOM 
Table 2. Thresholding parameter for proposed algorithm

\begin{tabular}{|c|c|c|c|}
\hline $\begin{array}{c}\text { Thresholding } \\
\text { Parameter }\end{array}$ & $\begin{array}{c}\text { Threshold-level (seed } \\
\text { point) }\end{array}$ & $\begin{array}{c}\text { Searching Region } \\
\text { (ROI) }\end{array}$ & Description \\
\hline $\begin{array}{l}\text { Intensity-based } \\
\text { thresholding }\end{array}$ & $\begin{array}{l}300<\mathrm{x}<2000 \\
(\mathrm{x}: \mathrm{HU} \text { value })\end{array}$ & $\begin{array}{l}\text { High Intensity region } \\
\text { (bone, stone, stent) }\end{array}$ & $\begin{array}{c}\text { To remove low intensity region } \\
\text { (stomach, gallbladder, intestines, liver, } \\
\text { pancreas and spleen, etc.) }\end{array}$ \\
\hline $\begin{array}{l}\text { Region-based } \\
\text { thresholding }\end{array}$ & $\begin{array}{c}80<\mathrm{x}<1,000,000 \\
(\mathrm{x}: \text { Area(no: pixels in a } \\
\text { region) })\end{array}$ & Bone region & $\begin{array}{l}\text { To remove very large and small region } \\
\text { (rib and spine group) }\end{array}$ \\
\hline $\begin{array}{l}\text { RVS-based } \\
\text { thresholding }\end{array}$ & $\begin{array}{c}2<\mathrm{x}<1 \\
(\mathrm{x}: \mathrm{RVS} \text { value })\end{array}$ & $\begin{array}{l}\text { True positive region } \\
\text { (bone) }\end{array}$ & To reduce false positive \\
\hline $\begin{array}{l}\text { SAV-based } \\
\text { thresholding }\end{array}$ & $\begin{array}{c}\mathrm{x}>0.2 \\
\text { (x: SAV ratio difference) }\end{array}$ & $\begin{array}{c}\text { True positive region } \\
\text { (bone) }\end{array}$ & To remove stent \\
\hline
\end{tabular}

file is started the segmentation process. Intensitybased thresholding: Because of distinct intensity range between the stone and other low-intensity organs, Otsu's thresholding becomes the optimal method for kidney stone segmentation. Large object with high intensity value (stone and bone) are easier to detect and segment compared with small ones with low intensity values. Therefore, stone and bone can extracted among other soft tissue and organs using Otsu's thresholding and morphological operation is also used to enhance the output image [29]. This segmentation can only remove low intensity region and the result is contained all high intensity region (stone, calcification, bones and stent) as shown in Fig. 3 (a).

Region-based thresholding: Kidney stones are available in a variety of sizes from the small-size as grain of sand until the large-size as golf balls. Bony skeleton (rib, vertebral column and pelvic cage) have large amount of voxel compare with other objects. Therefore, taking bony skeleton away from image is performed based on area-based thresholding. The area of each object can be calculated using Eq. (2). Moreover, because of the nature of CT scanning in kidney stone case, the location of kidney stone is enclosed inside of bony skeleton and bed-mat is existed at the behind of bony skeleton and stone. Estimation two coordinate thresholding values have been applied for locationbased thresholding algorithm [30]. As a result of the pre-processing, bony skeleton and some bone fragments are removed from the image. But, some noise from segmentation error, calcification and stent are left together with stone as shown in Fig. 3 (b). Therefore, false positive reduction is performed to distinguish the stone from other unwanted noise.

\subsection{False positive reduction}

Feature extraction: In this step, the basic 3D morphological features (area, volume and the surface area of stone object) are firstly computed from the output image of segmentation. Moreover, volume of sphere is also calculated for volume ratio measurement. As the effective and efficient features, Ratio Volume Sphere (RVS) and SAV ratio are formulated for noise removal.

Small Noise removing: A comparison between volume of object and volume of sphere, RVS, can predict the shape of 3D object. Mostly, the shapes of noises (non-stone objects) look like fiber-shaped appearance and their RVS values are between 1 and 2. Therefore, the fiber-shaped non-stone objects can be eliminated by using RVS based thresholding (with two threshold value, 1 and 2).

There are many varieties of shapes and sizes of kidney stones, such as rounded-shape, sphericalshape, oval-shape and staghorn-shape. Some stones often have unsmooth surface with branches, especially large stones. The shape of small stones is round, having no-branch and smooth surface. Therefore, the segmented object which is small and branch can be regarded as small noise (fiber-shaped noise). RVS can reduce fiber-shaped noise from the segmented output as shown in Fig. 3 (c).

Stents and large noise removing: In some cases, stent is remained as noise, after removing fibershaped non-stone object. It is difficult to eliminate the stent from bone and stone by using intensity and size features. So, the proposed study applied SAV based thresholding because of their shape differences. Although there is a slight different in SAV value among large noise and stent and stone, there is a specific gap when SAV of stone is compared with SAV of sphere (standard SAV). In this way, the stone can distinguish from the stent and large noise and then only the kidney stone is output as the final result in Fig. 3 (d).

\section{Experimental results}

Data analysis for this study was tested on abdominal CT images which were based on the 
incidence of kidney stone. The experiments were carried out on a PC with Intel® Core (TM).

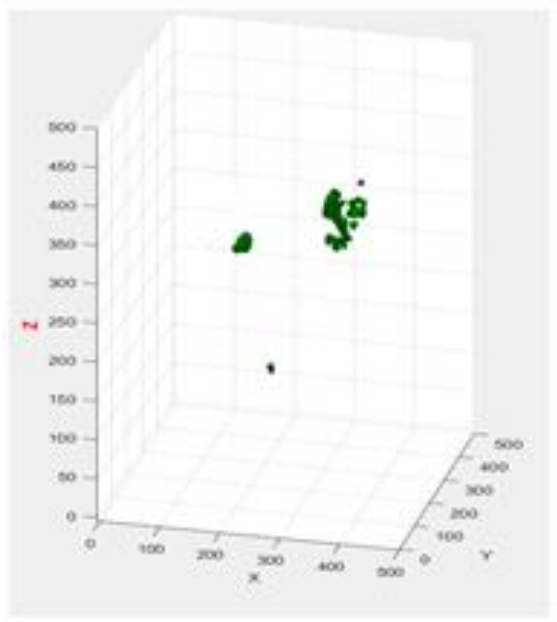

(a)

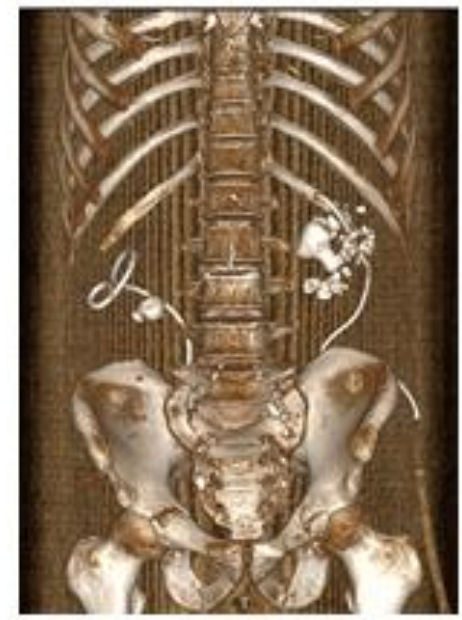

(b)

Figure. 4 3D visualization of segmented output using: (a) the proposed algorithm and (b) using RadiAnt DICOM Viewer (64-bit) application

\subsection{D visualization}

Visualization of CT data is a vital role of kidney stone diagnosing. In this study, an isosurfacematlab-function is used to extract isosurface-data from 3D volume array. Fig. 4 illustrated the visualized objects of the proposed algorithm by comparing the object of RadiAnt DICOM Viewer (64-bit) application. To view all sides of stone, the object can be rotated by dragging the mouse on the plane. Comparing the result of RadiAnt DICOM Viewer, the proposed algorithm can visualize the kidney stones clearly without any disturbances as shown in Fig. 4. This experiment shows that the proposed scheme can present 3D output image with highest clarity and lowest disturbance.

\subsection{Segmentation result}

We developed a fully automatic computer-aided diagnosis system to segment kidney stone on CT images. The proposed algorithm can detect 64 stones from 35 patients while RadiAnt DICOM Viewer can display 53 stones from the same database. The proposed algorithm can reduce many unwanted objects which impossible to remove by RadiAnt DICOM Viewer as shown in Fig. 4. Moreover, the number of stone segmented with proposed algorithm is increased from 53 to 64 compare with RadiAnt DICOM Viewer. Therefore, the segmentation result using proposed algorithm is 64 of true positive with 72 of false positive which is changed over18 \%difference compare with RadiAnt DICOM Viewer.

\subsection{Performance evaluation}

For performance evaluation, sensitivity was used in this study for a comparison of proposed algorithm. In [31], it is calculated using TP and FN rate as shown below:

$$
\text { Sensitivity }=\frac{T P}{(T P+F N)}
$$

TP is used for kidney stone, which are correctly detected as stone. FN is used for non-stone which are detected as stone. The program output and manual record were arranged to valid the performance of the proposed algorithm. The experimental result for performance evaluation is presented in Table 3 where sensitivity is calculated using Eq. (12). Three processes of the proposed algorithm were $47 \%, 64 \%$ and $70 \%$ in sensitivity, respectively. It is found that these methods extract the stone with a significant true positive (stone) detection. It gives a clear output image for stone diagnosis although there are still remain false negative.

The proposed algorithm presented two efficient methods (RVS and SAV ratio) to reduce the number of false positive. RVS reduces most of false positive (especially small fiber-shape non-stone objects) about $70 \%$ compare with the segmentation result. SAV ratio can extract the stent and large false positive among the stones that improve up to $70 \%$ of overall accuracy of system. 
Table 3. False positive reduction results

\begin{tabular}{lccc} 
& Segmentation result & RVS-based thresholding & SAV-based thresholding \\
\hline Sensitivity & $47 \%$ & $64 \%$ & $70 \%$ \\
True positive (TP) & 64 & 64 & 64 \\
False negative (FN) & 72 & 35 & 27 \\
\hline
\end{tabular}

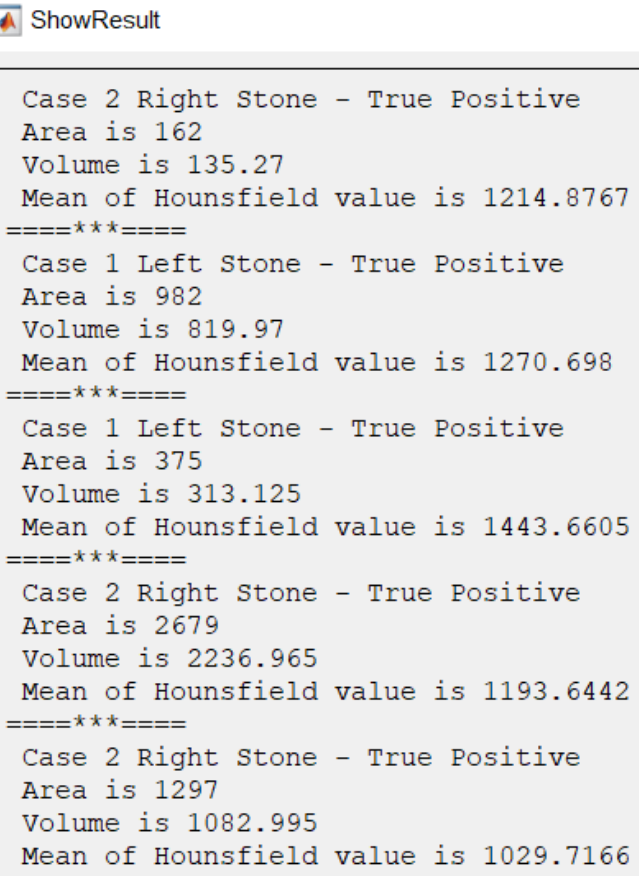

Figure. 5 Sample text output file for a patient

\subsection{Output information}

In additional 3D visualization, text file was generated by the proposed algorithm as output. The meaningful structural information of text file are included the location of stone (left, right or bladder), size of stone (area and volume) and average density of stone (Hounsfield unit) as shown in Fig. 5. The location of stone was estimated by using $\mathrm{X}, \mathrm{Y}$ and $\mathrm{Z}$ coordinate points. The area and volume were measured using Eqs. (2) and (3). And then, the average density of stone was calculated with the following;

$$
\text { Average } H U=\frac{\text { Total } H U \text { value of object }}{\text { Number of pixels of object }}
$$

\section{Conclusion and future work}

We developed a fully automatic computer-aided diagnosis system to segment kidney stone on CT images. Novelty of my proposed research is using segmentation on all slices of object and feature extraction on 3D matrix (3D morphological feature). Based on the visualization result, clarity of the output image of the proposed segmentation algorithm was higher than previous research and RadiAnt DICOM Viewer. Moreover, it can segment 64 kidney stones while RadiAnt DICOM Viewer segments 53 stones.

The majority of false positives reduction was due to similar shape, size and intensity of stone with noise. So, we also developed next two efficient features (RVS and SAV ratio features) to address the challenge of image noise in this setting. These two proposed features, RVS and SAV ratio are optimized a desirable property of a computer-aided diagnosis system by reducing image noise in the segmented result. According manual analysis, the segmented output contains two kinds of noise: fibershaped small noise and large noise which are difficult to distinguish with kidney stone. The optimal threshold range established using RVS feature is effective to extract the fiber-shaped noise among true positive stones. SAV feature is also important factor for large noise and stent removing because of a gap SAV difference between stone and non-stone. So, the proposed false reduction algorithm using RVS and SAV features can reduce the number of false negative from 72 to 27 as shown 
in Table 3. Although the proposed segmentation algorithm can segment some small stones that is impossible to display in RadiAnt DICOM Viewer, the accuracy of proposed segmentation was relatively low.

In conclusion, we have developed and validated an accurate method with estimation of coordinate points in the stone region which was measured independently by the expert radiologists. At 35 patients on the test dataset, the sensitivities of each process in Table 3 are $47 \%, 64 \%$ and $70 \%$, respectively. The method can also segment the kidney stone automatically, enabling false reduction based on 3D morphological feature. For CT scan interpretation, the method can provide the meaningful information such as stone location, stone size and stone density. This paper only showed extraction of 3D kidney stone object without any noise, but it is still weak in robustness. Therefore, we plan to explore more robust segmentation and feature extraction algorithm to develop the accurate method for stone diagnosis.

\section{References}

[1] M. Kantrowitz, "CancerPoints _ Diagnostic Imaging", $\quad$ www.cancerpoints.com, 2018. [Online]. Available: http://www.kantrowitz.com/cancerpoints/diagn osticimaging.html.

[2] W. Brisbane, M. R. Bailey, and M. D. Sorensen, "An overview of kidney stone imaging techniques", Nat. Rev. Urol., Vol. 13, No. 11, pp. 654-662, 2016.

[3] T. Heinonen, P. Dastidar, and H. Frey, "Applications of MR image segmentation," Int. J. Bioelectromagn., Vol. 1, No. 1, pp. 35-46, 1999.

[4] L. R. Varshney, "Abdominal organ segmentation in CT scan images: a survey", Semantic Scholar. ALLEN INSTITUTE for ARTIFICIAL INTELLIGENCE, pp. 1-4, 2005.

[5] V. C. Y. Tang and A. Attwell-Heap, "Computed tomography versus ureteroscopy in identification of renal tract stone with ureteral stent in situ", Ann. R. Coll. Surg. Engl., Vol. 93, No. 8, pp. 639-641, 2011.

[6] P. R. Tamiselvi, "Detection of renal Calculi Using Semi Automatic Segmentation Approach", Int. J. Eng. Sci. Innov. Technol., Vol. 2, No. 3, pp. 547-552, 2013.

[7] P. R. Tamilselvi and P. Thangaraj, "Computer aided diagnosis system for stone detection and early detection of kidney stones", J. Comput. Sci., Vol. 7, No. 2, pp. 250-254, 2011.
[8] M. Pathak, H. Sadawarti, and S. Singh, "Features extraction and classification for detection of kidney stone region in ultrasound images", Int. J. Multidiscip. Res. Dev., Vol. 3, No. 5, pp. 81-83, 2016.

[9] J. Verma, M. Nath, P. Tripathi, and K. K. Saini, "Analysis and identification of kidney stone using Kth nearest neighbour (KNN) and support vector machine (SVM) classification techniques", Pattern Recognit. Image Anal., Vol. 27, No. 3, pp. 574-580, 2017.

[10] P. R. Tamilselvi and P. Thangaraj, "Segmentation of Calculi from Ultrasound Kidney Images by Region Indicator with Contour Segmentation Method", Glob. J. Comput. Sci. Technol., Vol. 11, No. 22, 2011.

[11] K. Viswanath, R. Gunasundari, and S. A. Hussan, "VLSI Implementation and Analysis of Kidney Stone Detection by Level Set Segmentation and ANN Classification", Procedia Comput. Sci., Vol. 48, No. Iccc, pp. 612-622, 2015.

[12] G. Ravichandran and V. Palanivel, "NonLinear Enhancement and Selection of Plane for Segmenting the Abdominal Image for Kidney Stone Identification", Int. J. ofAdvanced Res. Electron. Commun. Eng., Vol. 5, No. 2, pp. 430-434, 2016.

[13] P. Akkasaligar, S. Biradar, and V. Kumber, "Kidney stone detection in Computed Tomorgrahy Images", In: Proc. of 2017 International Conference on Smart Technologies for Smart Nation, Vol. 6, No. 4, pp. 353-356, 2017.

[14] S. Ebrahimi and V. Y. Mariano, "Image Quality Improvement in Kidney Stone Detection on Computed Tomography Images", J. Image Graph., Vol. 3, No. 1, pp. 40-46, 2015.

[15] J. Liu, S. Wang, E. B. Turkbey, M. G. Linguraru, J. Yao, and R. M. Summers, "Computer-aided detection of renal calculi from noncontrast CT images using TV-flow and MSER features", Med. Phys., Vol. 42, No. 1, pp. 144-53, 2015.

[16] H. S. Lee, H. Hong, and J. Kim, "Detection and Segmentation of Small Renal Masses in Contrast- Enhanced Ct Images Using Texture and Context Feature Classification", In: Proc. of 2017 IEEE 14 th International Symposium on Biomedical Imaging, pp. 583-586, 2017.

[17] D. Khattab, H. M. Ebied, A. S. Hussein, and M. F. Tolba, "Color image segmentation based on different color space models using automatic GrabCut", Scientific World Journal, Vol. 2014, p. 126025, 2014. 
[18] S. Raut, M. Raghuvanshi, R. Dharaskar, and A. Raut, "Image segmentation - A state-of-art survey for prediction", In: Proc. Int. Conf. $A d v$. Comput. Control, pp. 420-424, 2009.

[19] S. Naz, H. Majeed, and H. Irshad, "Image segmentation using fuzzy clustering: A survey", In: Proc. of 2010 6th Int. Conf. Emerg. Technol., pp. 181-186, 2010.

[20] A. K. Chaubey, "Comparison of The Local and Global Thresholding Methods in Image Segmentation", World J. Res. Rev., Vol. 2, No. 1, pp. 1-4, 2016.

[21] O. Wirjadi, "Survey of 3d image segmentation methods", Kaiserslautern Deutschland, 2007.

[22] R. Srisha and M. Khan, "Morphological Operations for Image Processing: Understanding and its Applications Morphological Operations for Image Processing: Understanding and its Applications", NCVSComs-13, pp. 17-19, 2013.

[23] The Mathworks Inc., "Measure properties of image regions - MATLAB regionprops", MATLAB Documentation, 2013.

[24] X. Duan et al., "Kidney Stone Volume Estimation from Computerized Tomography Images Using a Model Based Method of Correcting for the Point Spread Function", $J$. Urol., Vol. 188, No. 3, pp. 989-995, 2012.

[25] D. Legland, K. Kiêu, and M.-F. Devaux, "Computation of Minkowski Measures on 2D and 3D Binary Images", Image Anal. Stereol., Vol. 26, No. 2, p. 83, 2011.

[26] "Surface-area-to-volume ratio - Wikipedia".

[27] W. Finch, R. Johnston, N. Shaida, A. Winterbottom, and O. Wiseman, "Measuring stone volume - Three-dimensional software reconstruction or an ellipsoid algebra formula?", BJU Int., Vol. 113, No. 4, pp. 610-614, 2014.

[28] T. Paulose, M. Montévil, L. Speroni, F. Cerruti, C. Sonnenschein, and A. M. Soto, "SAMA: A method for 3D morphological analysis", PLOS One, Vol. 11, No. 4, pp. 1-14, 2016.

[29] N. Thein, H. A. Nugroho, T. B. Adji, and K. Hamamoto, "An image preprocessing method for kidney stone segmentation in CT scan images", In: Proc. of CENIM 2018, 2018.

[30] N. Thein, K. Hamamoto, H. A. Nugroho, and T. B. Adji, "A comparison of three preprocessing techniques for kidney stone segmentation in CT scan images."

[31] N. Thein, K. Hamamoto, H. A. Nugroho, and T. B. Adji, "A comparison of three preprocessing techniques for kidney stone segmentation in CT scan images", In: Proc. of the 11th Biomedical Engineering International Conference, 2018. 